Article

\title{
Comparison of Random Forest, k-Nearest Neighbor, and Support Vector Machine Classifiers for Land Cover Classification Using Sentinel-2 Imagery
}

\author{
Phan Thanh Noi ${ }^{1,2, *}$ and Martin Kappas ${ }^{1}$ \\ 1 Cartography, GIS and Remote Sensing Department, Institute of Geography, University of Göttingen, \\ Goldschmidt Street 5, 37077 Göttingen, Germany; mkappas@gwdg.de \\ 2 Cartography and Geodesy Department, Land Management Faculty, Vietnam National University of \\ Agriculture, Hanoi 100000, Vietnam \\ * Correspondence: phan.thanh.noi@gmail.com; Tel.: +49-551-399-805
}

Received: 20 November 2017; Accepted: 20 December 2017; Published: 22 December 2017

\begin{abstract}
In previous classification studies, three non-parametric classifiers, Random Forest (RF), k-Nearest Neighbor $(\mathrm{kNN})$, and Support Vector Machine (SVM), were reported as the foremost classifiers at producing high accuracies. However, only a few studies have compared the performances of these classifiers with different training sample sizes for the same remote sensing images, particularly the Sentinel-2 Multispectral Imager (MSI). In this study, we examined and compared the performances of the RF, $\mathrm{kNN}$, and SVM classifiers for land use/cover classification using Sentinel-2 image data. An area of $30 \times 30 \mathrm{~km}^{2}$ within the Red River Delta of Vietnam with six land use/cover types was classified using 14 different training sample sizes, including balanced and imbalanced, from 50 to over 1250 pixels/class. All classification results showed a high overall accuracy (OA) ranging from $90 \%$ to $95 \%$. Among the three classifiers and 14 sub-datasets, SVM produced the highest OA with the least sensitivity to the training sample sizes, followed consecutively by RF and kNN. In relation to the sample size, all three classifiers showed a similar and high OA (over $93.85 \%$ ) when the training sample size was large enough, i.e., greater than 750 pixels/class or representing an area of approximately $0.25 \%$ of the total study area. The high accuracy was achieved with both imbalanced and balanced datasets.
\end{abstract}

Keywords: Sentinel-2; Random Forest (RF); Support Vector Machine (SVM); k-Nearest Neighbor $(\mathrm{kNN})$; classification algorithms; training sample size

\section{Introduction}

There is undoubtedly a high demand for land use/cover maps for the monitoring and management of natural resources, development strategies, and global change studies [1-4]. Land use/cover maps are one of the most important documents that provide information for various applications, such as land use policy development, ecosystem services, urban planning, conservation, agricultural monitoring, and land use/cover dynamic assessment [5-9].

Remote sensing satellite images are considered as one of the most important data sources for land use/cover mapping [10] due to their extensive geographical coverage at an efficient cost while providing irreplaceable information on the earth's surface [11]. Land use/cover maps are usually produced based on remote sensing image classification approaches [12-14]. However, the accuracy and processing time of land use/cover maps using remote sensing images is still a challenge to the remote sensing community [15].

Sentinel-2 is the latest generation Earth observation mission of the ESA (European Space Agency) designed for land and coastal applications, and it includes the identical Sentinel-2 A and Sentinel-2 B 
satellites which launched in June 2015 and March 2017, respectively [16]. Sentinel-2 remains active and enhances the mission of Landsat and SPOT (Systeme Probatoire d'Observation de la Terre) [17]. It is a system with a wide-swath, high spatial resolution (10-60 m), temporal resolution (ten days/five days for Sentinel-2 A, B/Sentinel-2 A + B), and multi-spectral (13 spectral bands) capabilities. It has also gained great attention in research due to its free access and global coverage. A wide range of applications have been studied with Sentinel-2 A (and Sentinel-2 simulation data), such as soil moisture mapping [18], mapping urban surface water bodies [19], forest stress monitoring [20], and quantifying above ground biomass [21]. Clevers et al. [22] used Sentinel-2 data for retrieving LAI and leaf and canopy chlorophyll content of potato crops. Particularly, in land use and land cover mapping, the practicality of Sentinel-2 has been tested and showed the high potential of application $[23,24]$. However, because it is a new type of satellite imagery, there are only a handful of studies using Sentinel-2 for land use/cover mapping, and thus more research is necessary to conduct and evaluate the usefulness of this imagery.

According to Lu and Weng [25], it is not only the imagery appropriateness but also the right choice of classification method that affects the results of land use/cover mapping. In literature, a variety of classification methods have been developed and tested for land use/cover mapping using remote sensing data [26-28]. These methods range from unsupervised algorithms (i.e., ISODATA or K-means) to parametric supervised algorithms (i.e., maximum likelihood) and machine learning algorithms such as artificial neural networks (ANN), k-Nearest Neighbors (kNN), decision trees (DT), support vector machines (SVM), and random forest (RF).

In the last decade, the nonparametric methods (machine learning based algorithms) have gained great attention of remote sensing based applications. To understand the rise of machine learning methods in land use/cover mapping, we searched the number of articles in the ISI Web of Knowledge (Indexes in SCI-EXPANDED and SSCI) with different keywords ("land cover"* AND "classification"* AND “Maximum Likelihood (/ Artificial Neural Networks/k-Nearest Neighbors/Decision Trees/Support Vector Machines/Random Forest)" during the timespan from 2007 to 2017.

Figure 1 shows that in this timespan, the use of SVM and RF classification algorithms increased significantly. The number of articles using MLC and ANN have fluctuated throughout the years, but has generally remained steady. In recent years $(2014,2015$, and 2017), there were a handful of studies using $\mathrm{kNN}$, however, we have not found any publications using $\mathrm{kNN}$ in 2016 with our searched keywords. It should be mentioned that in Figure 1, the high number of papers with the keyword MLC does not mean that there is much research using MLC for classification. In fact, most studies from our searched list used the MLC method as one of the criteria to compare to other machine learning algorithms $[11,29,30]$.

The results shown in Figure 1 reflect the studies in recent literature. Prasad et al. [31] stated that DT is too sensitive to small changes in the training dataset. They also reported that DT is occasionally unstable and tends to overfit in the model. The ideal value of $\mathrm{k}$ for the $\mathrm{kNN}$ classifier is difficult to set [32]. The ANN method contains a high level of complexity in computational processing, causing it to become less popular in remote sensing based classification applications. SVM and RF are insensitive to noise or overtraining, which shows their ability in dealing with unbalanced data [33]. Therefore, among the nonparametric methods, SVM and RF are becoming increasingly popular in image classification studies [34].

Several studies have been implemented in order to find the best classification algorithm for land use/cover studies by comparing the performance of these classifiers either among themselves or with other classification algorithms. However, their conclusions are quite different. For example, in the studies by Adam et al. [34] and Ghosh and Joshi. [35], SVM and RF showed similar results of classification. Khatami et al. [11] found that SVM, kNN, and RF generally outperform other traditional supervised classifiers. Pouteau et al. [36] compared 6 machine learning algorithms (SVM, Naïve Bayes, C4.5, RF, Boosted Regression Tree, and kNN) with 6 satellite data sets from different sensors (Landsat-7 
ETM+, SPOT, AirSAR, TerraSAR-X, Quickbird, and WorldView-2) for Topical Ecosystems Classification and stated that kNN better performs for the Landsat-7 ETM+ classification. Most recently, Heydari and Mountrakis [37] studied the effects of the classifier selection, reference sample size, reference class distribution, and scene heterogeneity in per-pixel classification accuracy using 26 Landsat sites with five classification algorithms (Naïve Bayesian, kNN, SVM, Tree ensemble, and Artificial Neural Network). They concluded that SVM and kNN were the best classification methods for Landsat classification.

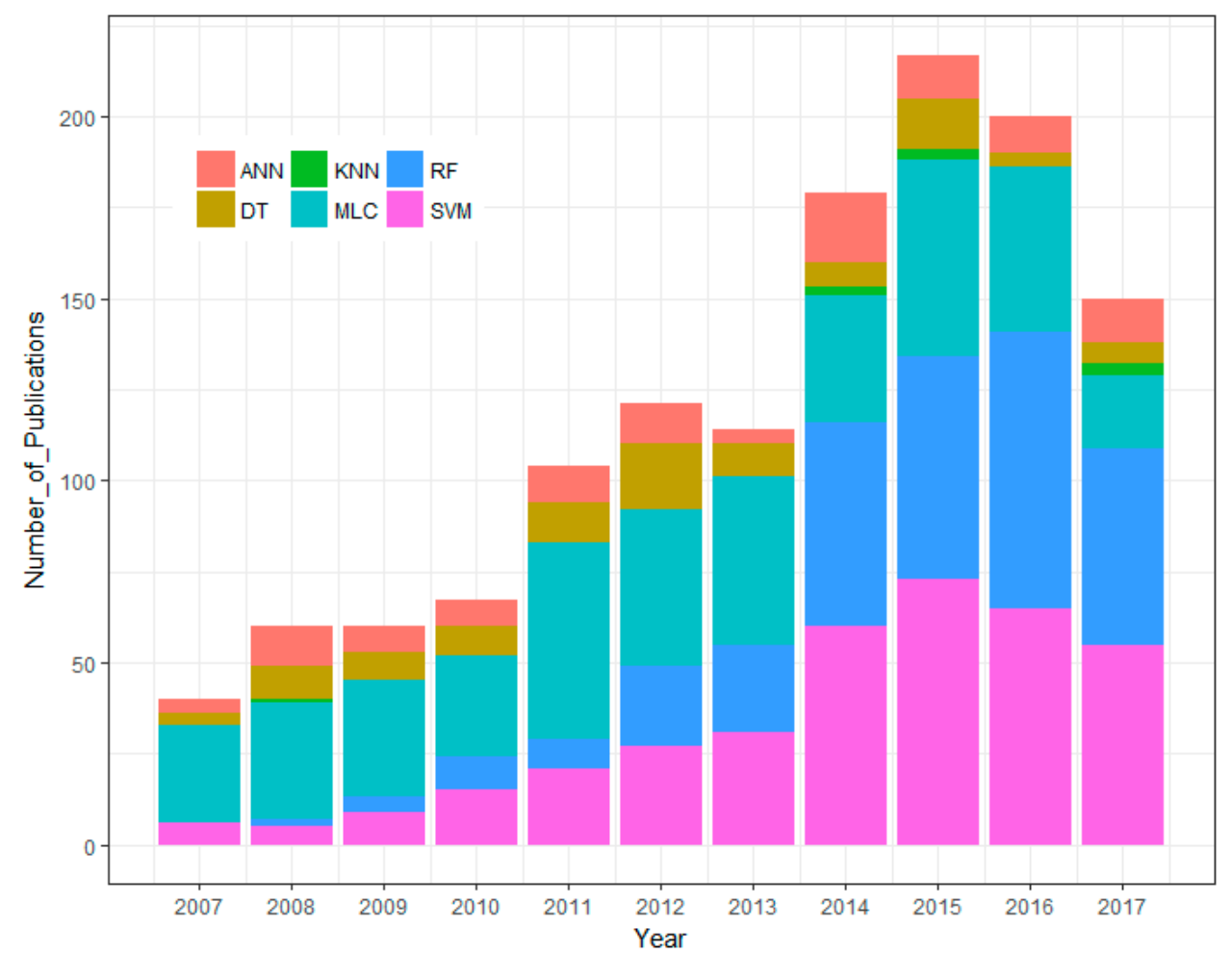

Figure 1. Number of articles in the ISI Web of Knowledge for a general search on different keywords in the last decade (2007-2017*). *Accessed on 13 August 2017.

In addition, to the best of our knowledge, only a limited amount of research was published that compared and evaluated the performance of RF, SVM, and kNN with different training sample strategies using Sentinel-2 imagery, especially in Vietnam. Therefore, it is practical for a study to compare and evaluate the performance of RF, SVM and kNN for land use/cover mapping over North Vietnam using the new satellite data, Sentinel-2 A. The objectives of this study are: (i) to evaluate the performance of the three most increasing classifiers, RF, kNN, and SVM, when applied to a Sentinel-2 image and (ii) to assess the effects of the training samples size, strategies, and type (balanced/imbalanced) on the accuracy of the classification results of the three aforementioned classifiers.

\section{Materials and Methods}

The overall methodology of the study is described in Figure 2. To fulfill the study objectives, a study area was selected based on the land cover characteristics and the availability of remote sensing imagery data. The remote sensing image was preprocessed, atmospherically corrected, and clipped to the study area (Figure 3). 


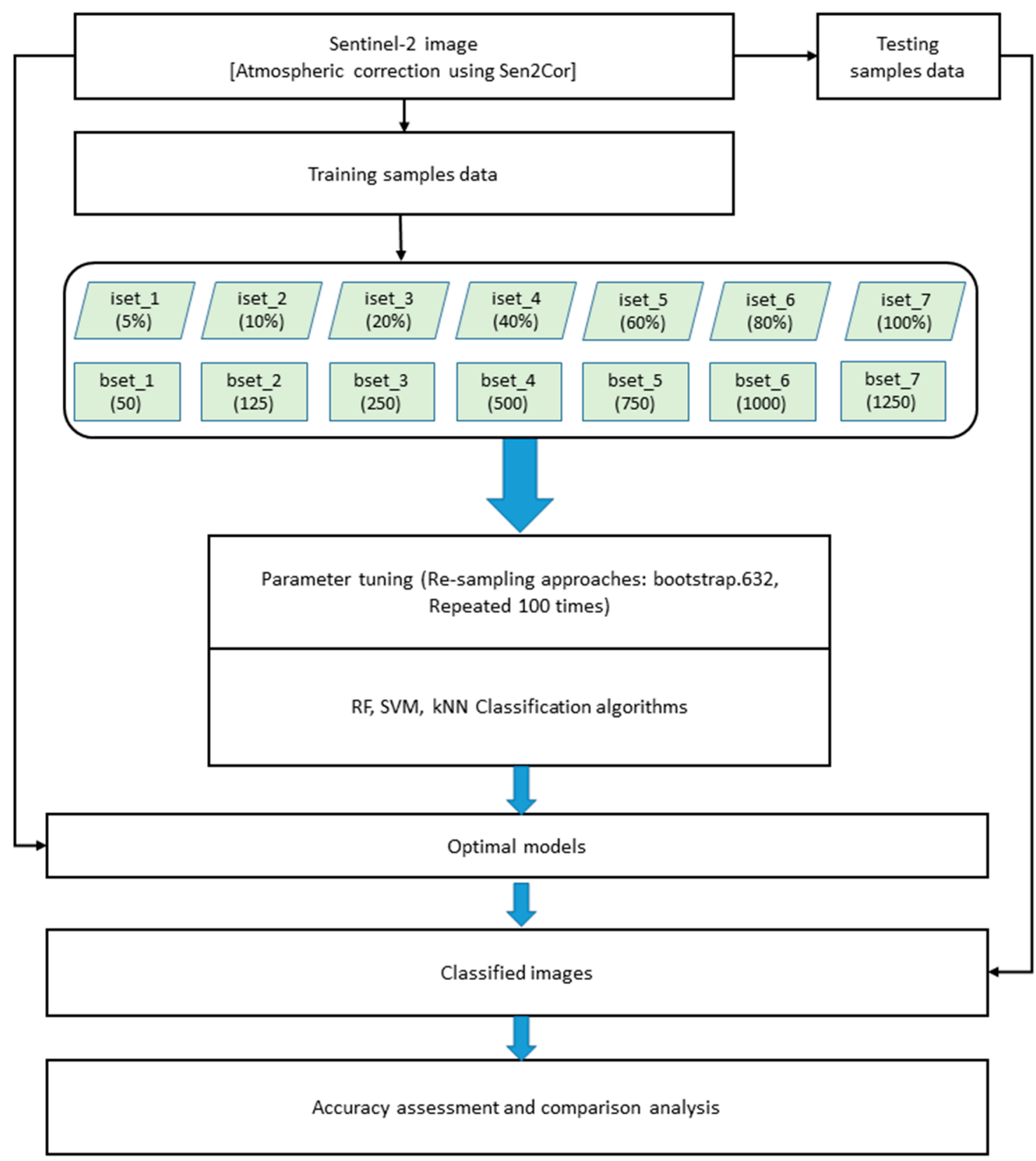

Figure 2. Flowchart of the study methods.

\subsection{Study Area}

In this study, in order to compare the performance of different classification algorithms on different data training sample strategies, an area of $30 \times 30 \mathrm{~km}^{2}$ of a peri-urban and rural with heterogeneous land cover area in the north of the Red River Delta (RRD), Vietnam was chosen (Figure 3). This is a typical land use/cover of the RRD area, slightly sloping from the southwest to the northeast. The study area mainly includes six typical classes: resident (fragment and distribution over the study area), impervious surface (including factory, block building and transportation, roads), agriculture, bare land, forest, and water. 

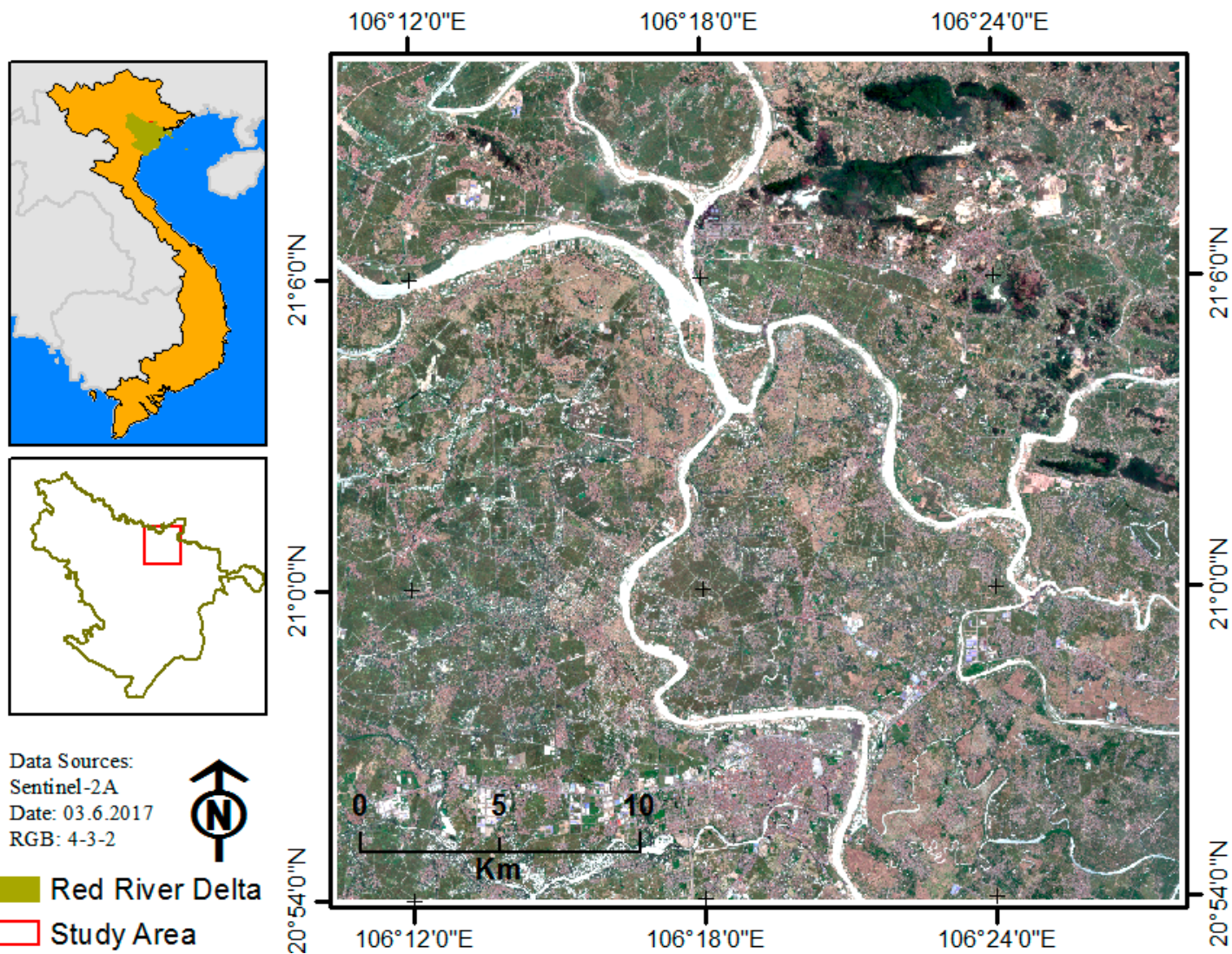

Figure 3. Location of the study area in the north of the Red River Delta (RRD), Vietnam.

\subsection{Data Used}

Sentinel-2 A, level 1 C (ID = L1C_T48QXJ_A010168_20170603T034152) acquired on 3 June 2017, was downloaded from the United States Geological Survey (USGS) website [38]. Spectral bands of the Sentinel-2 A satellite imagery are shown in Table 1 . The imagery was atmospherically corrected using the Sen2cor tool, which is available in the Sentinel Application Platform (SNAP) toolbox [22,39]. After atmospheric correction, 10 bands (2-8, 8A, 11 and 12) were composited with $20 \mathrm{~m}$ resolution and clipped to the study area $\left(30 \times 30 \mathrm{~km}^{2}\right)$. The composited-10 band-20m imagery was used for classification in this study.

Table 1. Spectral bands of the Sentinel-2 A satellite imagery.

\begin{tabular}{cccc}
\hline Spectral Band & Center Wavelength $(\mathbf{n m})$ & Band Width $(\mathbf{n m})$ & Spatial Resolution $(\mathbf{m})$ \\
\hline Band 1 & 443 & 20 & 60 \\
Band 2 & 490 & 65 & 10 \\
Band 3 & 560 & 35 & 10 \\
Band 4 & 665 & 30 & 10 \\
Band 5 & 705 & 15 & 20 \\
Band 6 & 740 & 15 & 20 \\
Band 7 & 783 & 20 & 20 \\
Band 8 & 842 & 115 & 10 \\
Band 8a & 865 & 20 & 20 \\
Band 9 & 945 & 20 & 60 \\
Band 10 & 1380 & 30 & 60 \\
Band 11 & 1610 & 90 & 20 \\
Band 12 & 2190 & 180 & 20 \\
\hline
\end{tabular}




\subsection{Training and Testing Sample Datasets}

The training data (training and testing samples) was collected based on the manual interpretation of the original Sentinel-2 data and high-resolution imagery available from Google Earth.

To collect training sample data, the create polygon tool in the ArcGIS 10.5 toolbox was used to create 135 polygons for each land cover class. Due to the different polygon sizes, the number of pixels for each land cover class also differed (Table 2).

Table 2. Training and testing sample sizes used in this study.

\begin{tabular}{ccc}
\hline Land Cover & Training (polygon/pixels) & Testing (pixels) \\
\hline Residential & $135 / 1410$ & 625 \\
Impervious surface & $135 / 1645$ & 427 \\
Agriculture & $135 / 2619$ & 614 \\
Bare land & $135 / 1274$ & 605 \\
Forest & $135 / 1267$ & 629 \\
Water & $135 / 1704$ & 628 \\
\hline
\end{tabular}

For an accurate assessment of the classification results, 650 points for every land cover class was collected. However, to ensure that the training and testing datasets were independent, we buffered $15 \mathrm{~m}$ for all point samples (testing dataset) and removed points which had buffered-points intersecting with (or belonging to) polygon samples. As results, we obtained the number of testing points (pixels) as shown in Table 2.

To evaluate the effect of the training sample sizes, as well as the performance of classification algorithms on the classification accuracies, we randomly divided training sample data into 14 different datasets (Table 3), in which seven-imbalanced datasets (iset_1, iset_2, iset_3, iset_4, iset_5, iset_6, and iset_7) had the corresponding sizes of 5\%,10\%,20\%,40\%,60\%,80\%, and 100\% of the total training data. The create Data Partition function in the caret package [40] was used to guarantee that all datasets had the same proportion-training sample of each land cover class. The remaining seven-balanced datasets were created as follows: bset_1 (50 pixels/class), bset_2 (125 pixels/class), bset_3 (250 pixels/class), bset_4 (500 pixels/class), bset_5 (750 pixels/class), bset_6 (1000 pixels/class), and bset_7 (1250 pixels/class). The number of pixels in each class for every sub-dataset was chosen to keep the most consistent size between the imbalanced and balanced training sample size; for example, the lowest number of pixels among the 6 land cover classes was 1267 pixels, leading bset_ 7 to have 1250 pixels for the balanced data for each class.

Table 3. Samples data for training classification.

\begin{tabular}{cccccccc}
\hline Imbalanced_data & iset_1 & iset_2 & iset_3 & iset_4 & iset_5 & iset_6 & iset_7 \\
\hline No. pixels & $5 \%$ & $10 \%$ & $20 \%$ & $40 \%$ & $60 \%$ & $80 \%$ & $100 \%$ \\
\hline Balanced data & bset_1 & bset_2 & bset_3 & bset_4 & bset_5 & bset_6 & bset_7 \\
\hline No. pixels & 50 & 100 & 250 & 500 & 750 & 1000 & 1250 \\
\hline
\end{tabular}

\subsection{Classification Algorithms and Tuning Parameters}

Tuned parameters play an important role in producing high accuracy results when using SVM, RF, and kNN. Each classifier has different tuning steps and tuned parameters. For each classifier, we tested a series of values for the tuning process with the optimal parameters determined based on the highest overall classification accuracy. In this study, the classified results under the optimal parameters of each classifier were used to compare the performance of classifiers [41]. 


\subsubsection{Support Vector Machine (SVM)}

In land cover classification studies, according to Knorn et al. [42] and Shi and Yang. [43], the radial basis function (RBF) kernel of the SVM classifier is commonly used and shows a good performance. Therefore, we used the RBF kernel to implement the SVM algorithm. There are two parameters that need to be set when applying the SVM classifier with RBF kernel: the optimum parameters of cost (C) and the kernel width parameter $(\gamma)$ [41,44]. The C parameter decides the size of misclassification allowed for non-separable training data, which makes the adjustment of the rigidity of training data possible [45]. The kernel width parameter $(\gamma)$ affects the smoothing of the shape of the class-dividing hyperplane [46]. Larger values of $C$ may lead to an over-fitting model [35], whereas increasing the $\gamma$ value will affect the shape of the class-dividing hyperplane, which may affect the classification accuracy results [35,47]. Following the study of Li et al. [28] and pretested to our dataset, in this study, to find the optimal parameters for SVM, ten values of $C\left(2^{-2}, 2^{-1}, 2^{0}, 2^{1}, 2^{2}, 2^{3}, 2^{4}, 2^{5}, 2^{6}, 2^{7}\right)$, and ten values of $\gamma\left(2^{-5}, 2^{-4}, 2^{-3}, 2^{-2}, 2^{-1}, 2^{0}, 2^{1}, 2^{2}, 2^{3}, 2^{4}\right)$ were tested. This procedure was applied to all 14 sub-datasets.

\subsubsection{Random Forest (RF)}

In order to implement the RF [33], two parameters need to be set up: the number of trees (ntree) and the number of features in each split (mtry). Several studies have stated that the satisfactory results could be achieved with the default parameters [12,48-50]. However, according to Liaw \& Wiener [48], the large number of trees will provide a stable result of variable importance. In addition, Breiman [33] stated that using more than the required number of trees may be unnecessary, but this does not harm the model. In addition, Feng et al. [51] stated that with ntree $=200$, RF could achieve accurate results. Regarding the mtry parameter, there are many studies that use the default value mtry $=\sqrt{ } \mathrm{p}$, where $p$ is the number of predictor variables [12]. However, in this study, to find the optimal RF model for classification, a range of values for both parameters were tested and evaluated: ntree $=100,200,500$, and 1000; $m$ try $=1: 10$ with a step size of 1 .

\subsection{3. k-Nearest Neighbor ( $\mathrm{kNN})$}

The kNN approach is a non-parametric [52] that has been used in the early 1970's in statistical applications [53]. The basic theory behind $\mathrm{kNN}$ is that in the calibration dataset, it finds a group of $\mathrm{k}$ samples that are nearest to unknown samples (e.g., based on distance functions). From these k samples, the label (class) of unknown samples are determined by calculating the average of the response variables (i.e., the class attributes of the $\mathrm{k}$ nearest neighbor) $[54,55]$. As a result, for this classifier, the $\mathrm{k}$ plays an important role in the performance of the $\mathrm{kNN}$, i.e., it is the key tuning parameter of $\mathrm{kNN} \mathrm{[41].}$ The parameter $\mathrm{k}$ was determined using a bootstrap procedure. In this study, we examined $\mathrm{k}$ values from 1 to 20 to identify the optimal $\mathrm{k}$ value for all training sample sets.

\subsection{Accuracy Assessment and Comparisons}

In order to assess the accuracy of classification performance, there are many metrics available in the literature. The two most popular metrics are overall accuracy (OA) and Kappa statistic. Recently however, the Kappa statistic is becoming less common in remote sensing classification accuracy assessment [37]. One of the drawbacks of solely using the OA metric is that it does not show the specific performance of classes. He and Garcia [56] stated that if input datasets (training samples) are highly imbalanced, the OA value might be deceiving, because the rare classes may be classified very poorly. He and Garcia [56] also suggested that when choosing OA as the criterion metric, the best class distribution is followed by the naturally occurring.

In this study, we used a stratified sampling approach; moreover, we divided training data into several sub-datasets, including imbalanced and balanced datasets. This approach matches the OA metric. In addition, to compare the performance of each classifier, we used the same training (input) 
and testing (validation) datasets; thus, the effect of individual class's distribution on OA does not bias the results. We also calculated the $95 \%$ confidence interval (error tolerance) $\delta$ of the probability estimate [57] for every OAs. Because we used the same testing datasets for all classification accuracy assessment, thus the $\delta$ were not significant different. (The detail of OAs and $\delta$ are shown in Appendix B). Therefore, to assess and compare the performance of the classifiers and the different datasets, we used overall accuracy (OA) as the criterion. In total, we have seven imbalanced datasets, seven balanced datasets, and three classifiers. Consequently, each classifier had 14 classification results, totaling 42 overall classification results.

\section{Results}

\subsection{The Effects of Tuning Parameters on Classification Accuracies}

Due to the limitation of space and the consistency of the results, only the results of eight sub-datasets—4 balanced (bset_1, bset_3, bset_5, bset_7) and 4 imbalanced (iset_1, iset_3, iset_5, iset_7) - are shown. The results of the remaining sub-datasets were still tested and showed a consistent trend in results.

\subsubsection{The kNN Classifier}

With the kNN classifier, to classify one object, the algorithm bases the class attributes of its $\mathrm{k}$ nearest neighbors [41]. Therefore, the $\mathrm{k}$ value plays an important role in the performance of $\mathrm{kNN}$, and is the key tuning parameter of $\mathrm{kNN}$ algorithm. In this study, we tested a range of k values (1 to 20) for choosing the optimal parameter of the $\mathrm{kNN}$ classifier using different sub-datasets.

Figure 4 shows the results of the kNN classifier error when applied to different sub-datasets. The lowest error was achieved with $\mathrm{k}=1$ for all datasets. As shown in Figure 4, when $\mathrm{k}$ increases from 1 to 20 , the error of the kNN classifier subsequently increases. This finding is consistent with the study by Qian et al. [46]. Therefore, the optimal k for the kNN classifier was chosen as k $=1$.
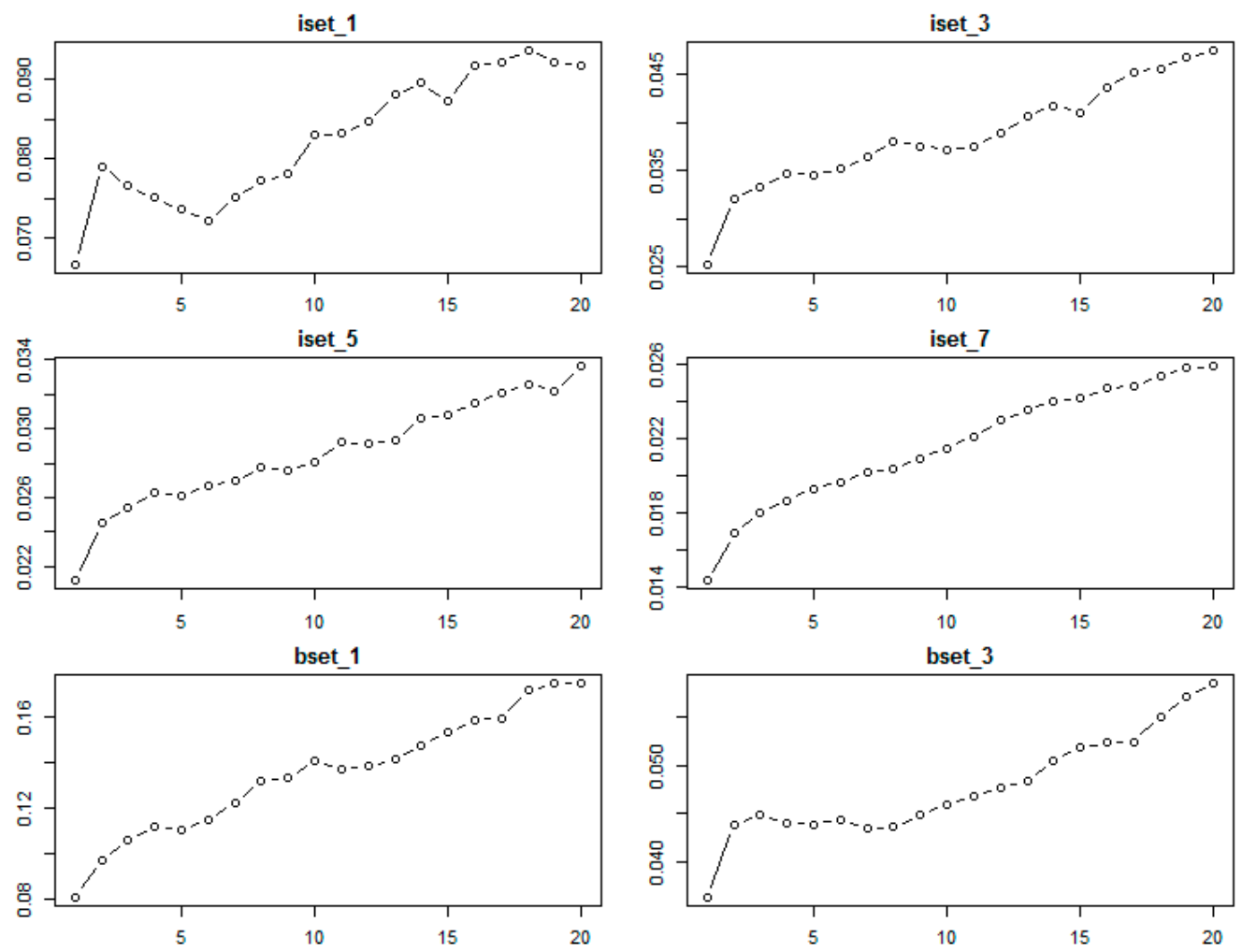

Figure 4. Cont. 

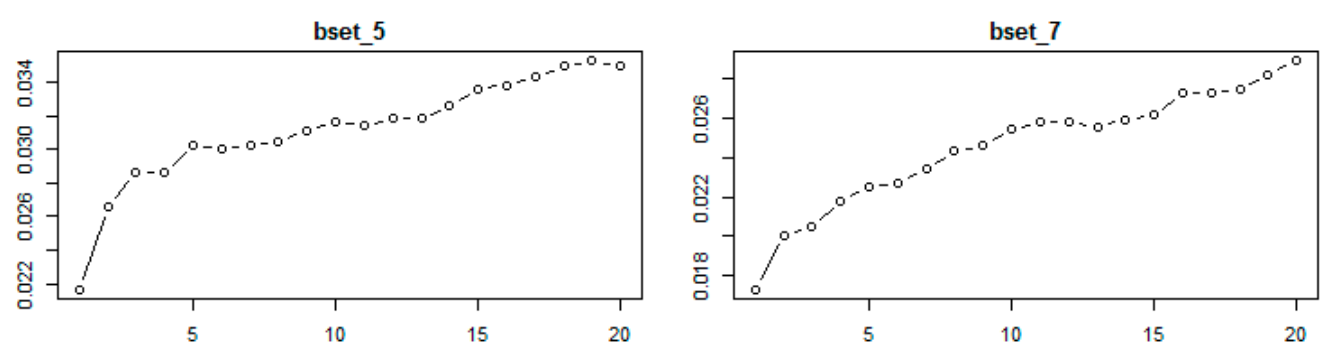

Figure 4. The relationship between classification error ( $y$-axis) and $\mathrm{k}$ value ( $x$-axis) parameters (1 to 20$)$ of the kNN classifier obtained from the bootstrap resampling approach using different sub-datasets of training sample data.

\subsubsection{The RF Classifier}

As stated in Section 2.4.2, there are two parameters that significantly affect the performance of the RF classifier: ntree and mtry. In this study, we used Sentinel-2 with ten bands for classification, meaning the input data has 10 variables. To find the optimal parameters for the RF classifier, several values (mtry $=1: 10 ;$ ntree $=100,200,500$, and 1000) were tested for all 14 sub-datasets. The highest results for all sub-datasets were obtained with mtry equal to 2, 3, or 4 (Figure 5).

As shown in Figure 5, when the mtry was 2, 3, or 4, the results of ntree 200, 500, and 1000 were similar. In addition, Figure 6 shows that out-of-bag (OOB) error decreased sharply when ntree increased from 1 to 100 . When ntree increased from 101 to 400, different sub-datasets had slightly different trends, however, generally, the OOBs were slightly reduced at all sub-datasets. All OOBs of all sub-datasets were almost remain stable when ntree increase from 400 to 500 . Therefore, ntree $=500$ was used as the optimal value for RF classifiers. The mtry value was chosen based on the highest results of mtry $=2,3$, or 4 .
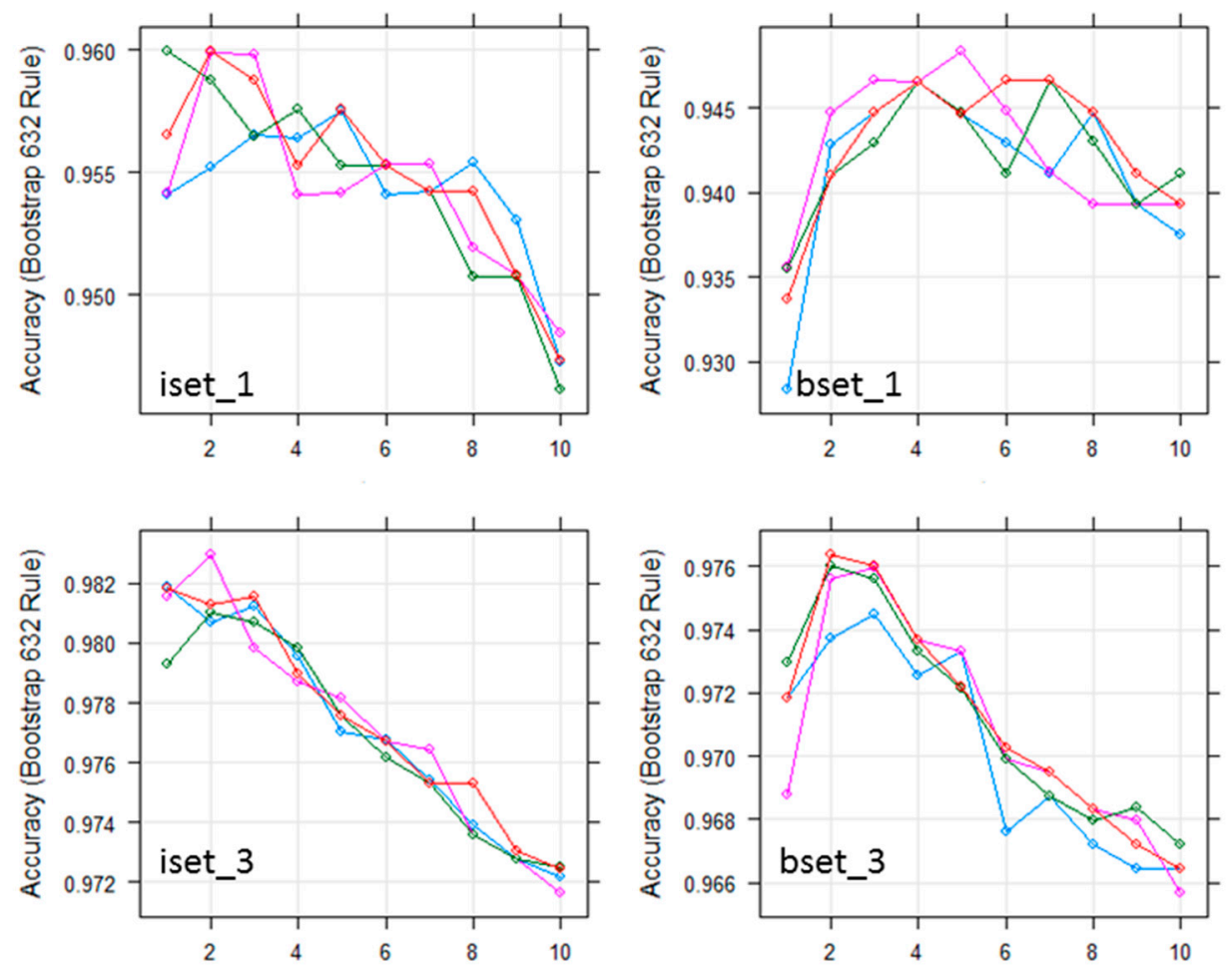

Figure 5. Cont. 

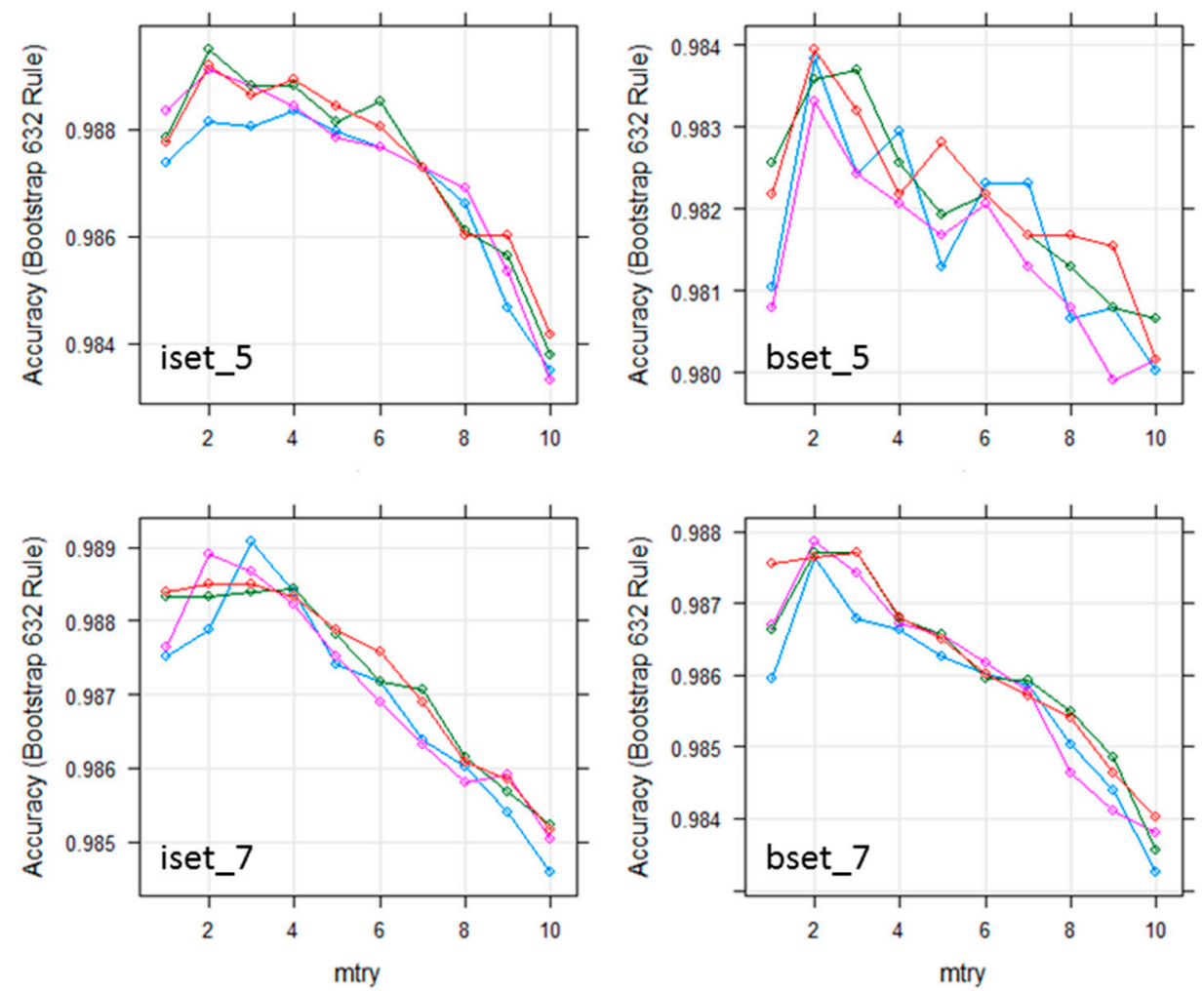

100

ntree

500 ○

1000

Figure 5. Effect of the number of trees and the number of random split variables at each node (mtry) on the overall accuracy for RF classification using all training sample data.
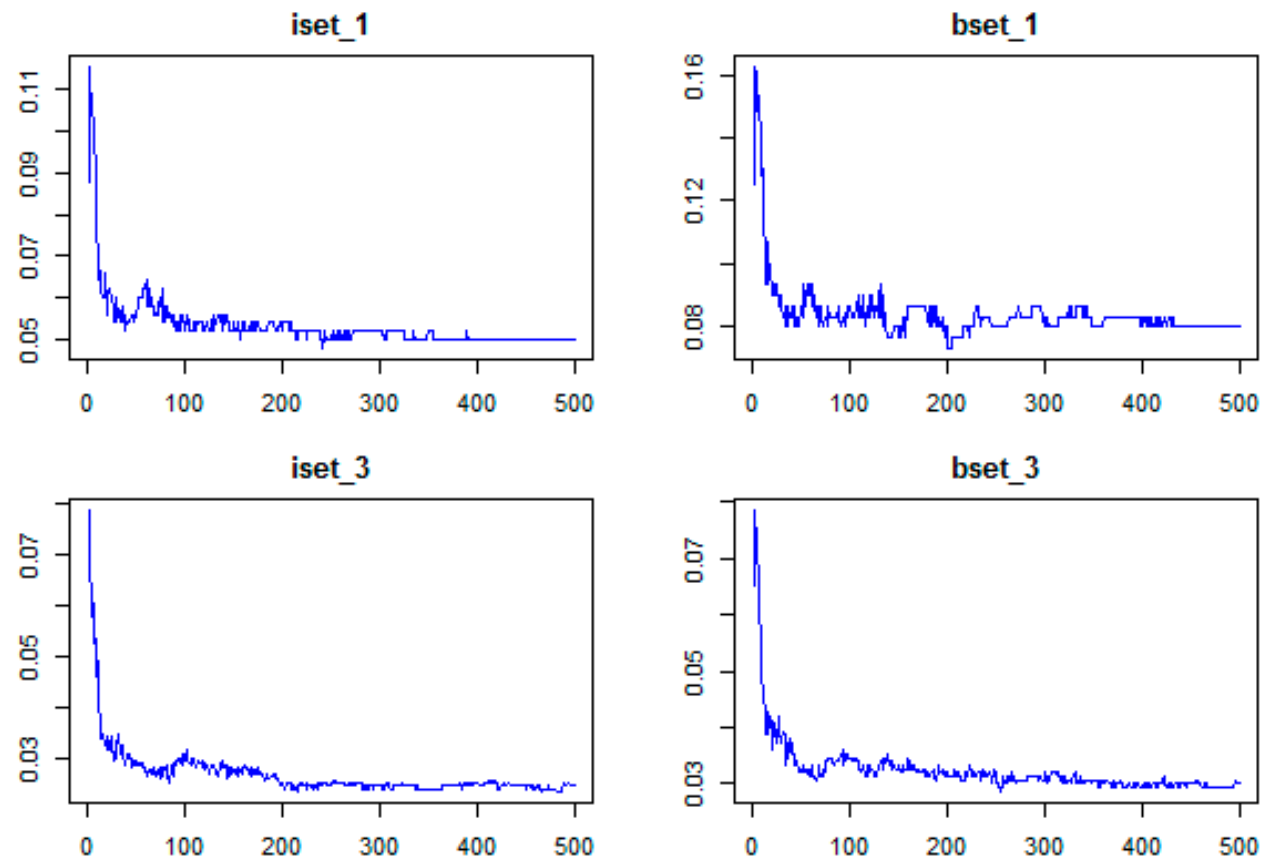

Figure 6. Cont. 

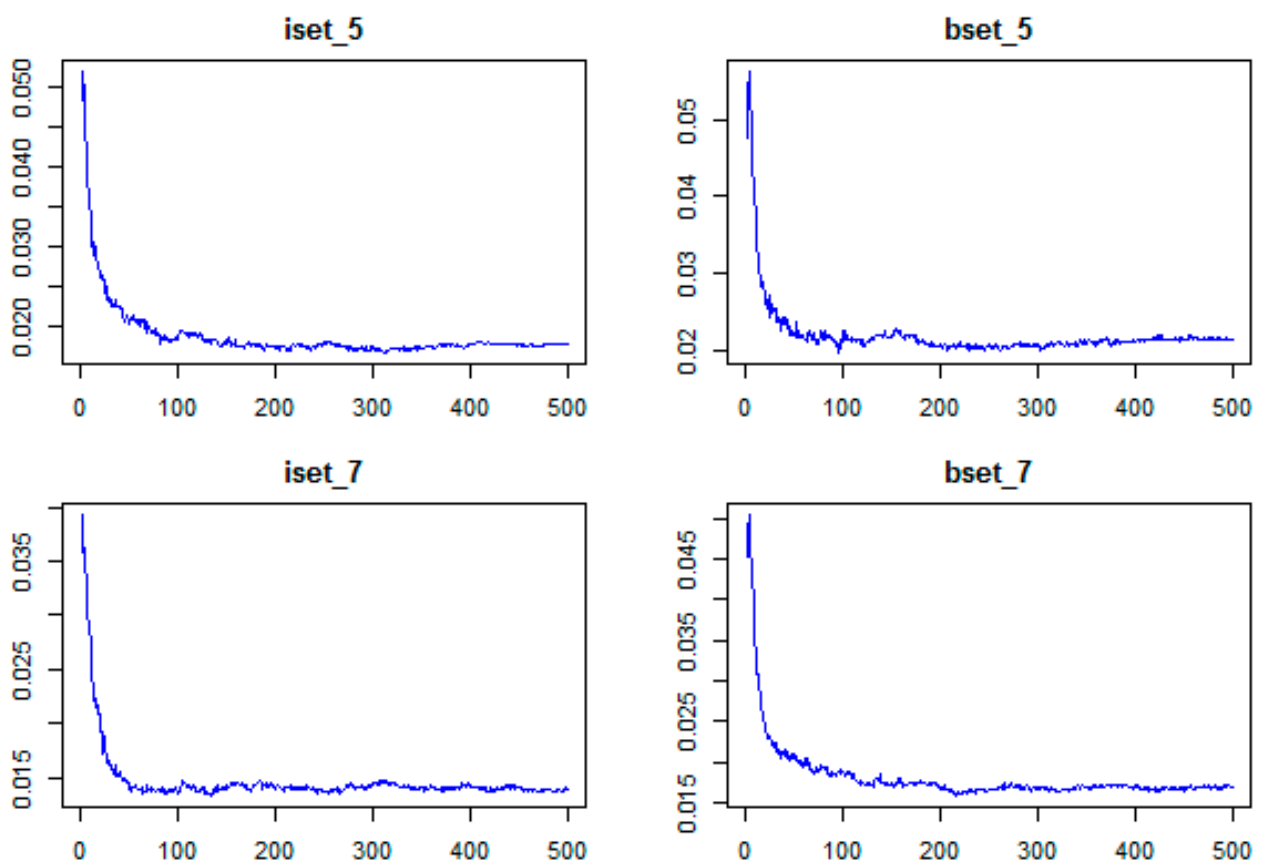

Figure 6. The relationship between OOB error ( $y$-axis) and ntree parameter ( $x$-axis) of the RF classifier using different sub-datasets of training sample data.

\subsubsection{The SVM Classifier}

In order to find the optimal values for the SVM model, several values were examined for $\mathrm{C}$ and $\gamma$ : $C\left(2^{-2}, 2^{-1}, 2^{0}, 2^{1}, 2^{2}, 2^{3}, 2^{4}, 2^{5}, 2^{6}, 2^{7}\right), \gamma\left(2^{-5}, 2^{-4}, 2^{-3}, 2^{-2}, 2^{-1}, 2^{0}, 2^{1}, 2^{2}, 2^{3}, 2^{4}\right)$.

Figure 7 shows the relationship between the OOB error and the SVM parameters. Based on these results, the optimal parameters of the 14 sub-datasets were chosen according to the lowest OOB error for each result. In general, a high value of $C$ and a low value of $\gamma$ produced the lowest error. The higher error was observed with a low $C$ value with both high (greater than eight) and low (less than 0.125) value of $\gamma$ (Figure 7). The optimal parameters (C and $\gamma$ ) of different sub-datasets varied (Table 4).

The optimal models (using optimal parameters) were applied to the whole image for classification results.
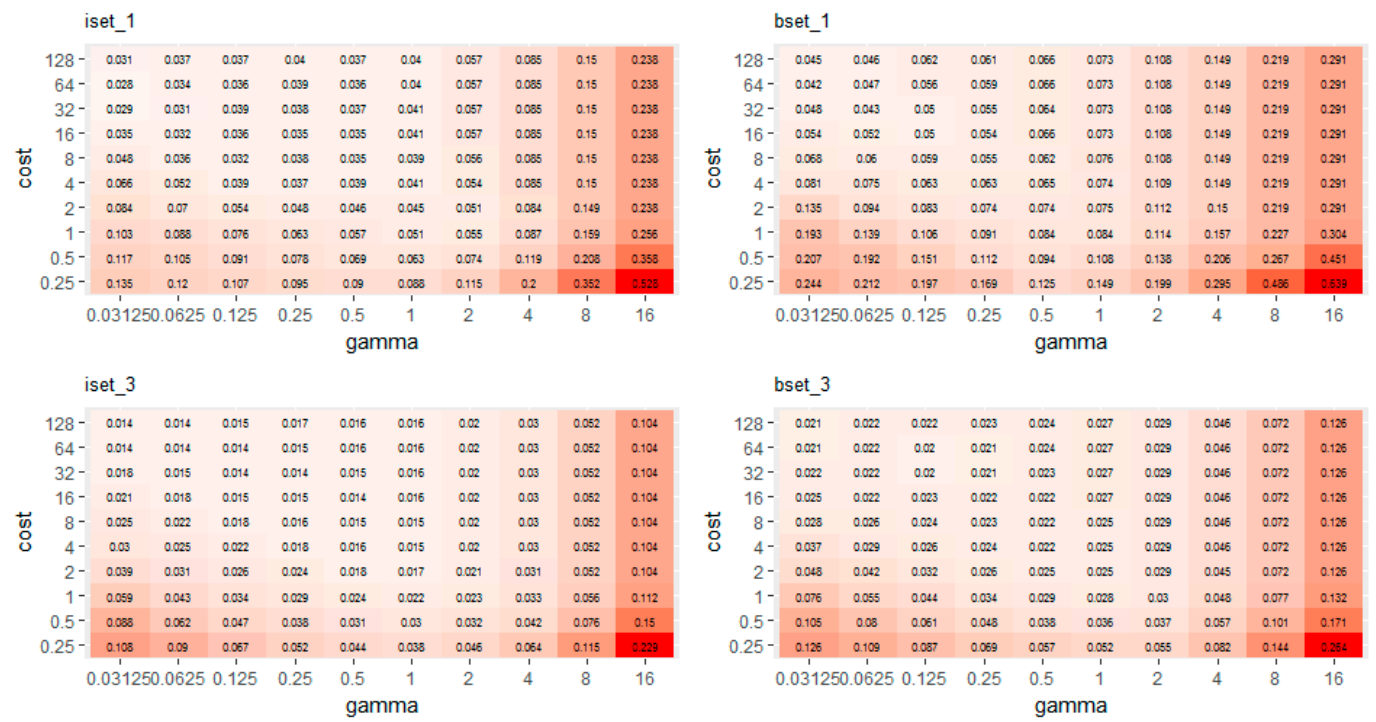

Figure 7. Cont. 

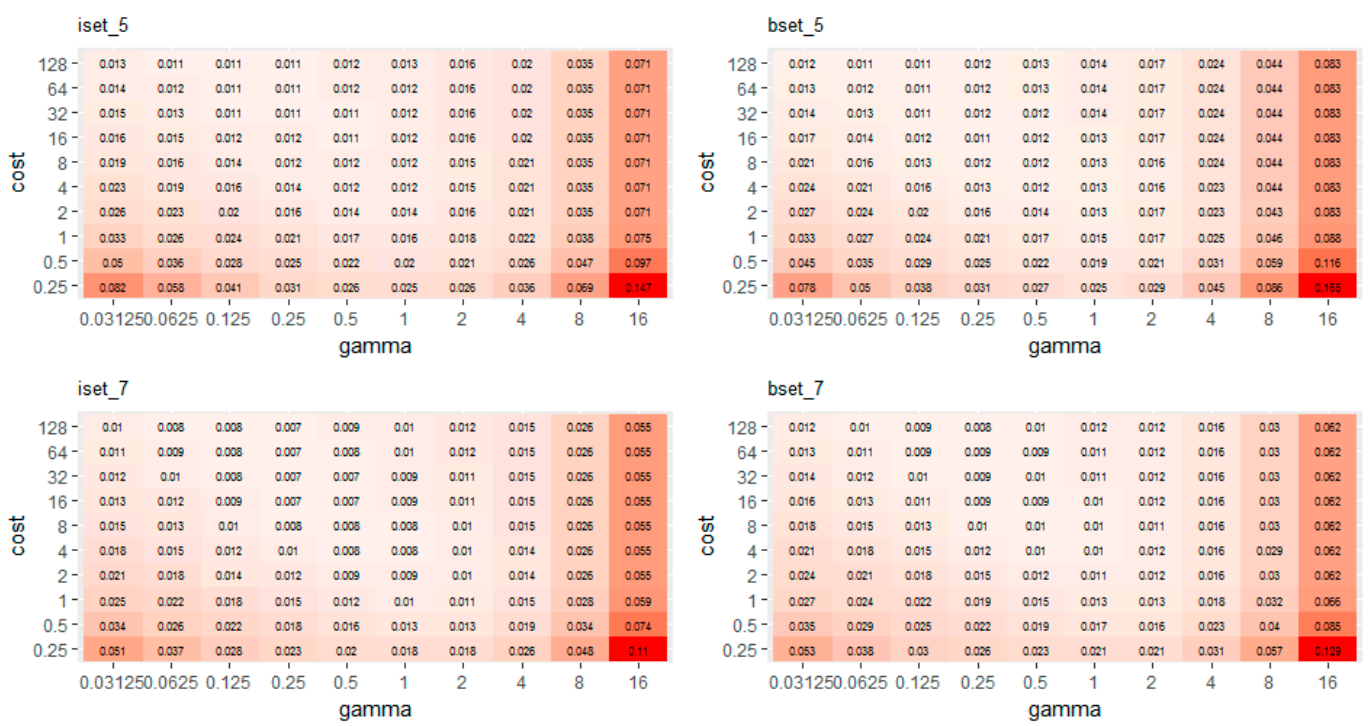

Figure 7. The relationship between classification error and parameters ( $C$ and $\gamma$ ) of the SVM classifier obtained from different sub-datasets of training sample data.

Table 4. The optimal parameters ( $\mathrm{C}$ and $\gamma)$ of different sub-datasets varied.

\begin{tabular}{cccccc}
\hline Imbalanced Dataset & $\gamma$ & C & Balanced Dataset & $\gamma$ & C \\
\hline iset_1 & 0.03125 & 64 & bset_1 & 0.03125 & 64 \\
iset_2 & 0.5 & 32 & bset_2 & 0.125 & 32 \\
iset_3 & 0.03125 & 128 & bset_3 & 0.125 & 64 \\
iset_4 & 0.25 & 32 & bset_4 & 0.125 & 64 \\
iset_5 & 0.125 & 64 & bset_5 & 0.125 & 128 \\
iset_6 & 0.5 & 32 & bset_6 & 0.25 & 64 \\
iset_7 & 0.25 & 64 & bset_7 & 0.25 & 128 \\
\hline
\end{tabular}

\subsection{The Performance of Different Classifiers on Imbalanced Datasets}

As shown in Figure 8, with all seven sub-datasets (iset_1 to iset_7), SVM always showed the most accurate results, followed by RF and kNN. However, the three highest accuracies of all classifiers were only slightly different. The accuracy results of SVM were not significantly different among different training sample sizes; from iset_1 to iset_7, the lowest was iset_1 data at $93.76 \%$ and the highest was iset_ 5 data at $95.32 \%$. In contrast, the classification accuracy of KNN and RF were significantly different between small sample sizes (iset_1) and large sample sizes (iset_7). With small and imbalanced training samples (iset_1, iset_2, and iset_3), there is a difference between classification accuracies. SVM produced a significantly higher accuracy than that of RF and kNN. This is consistent with the results reported from Shi and Yang [43]. From a small sample size (iset_1) to a larger sample size (iset_7), the accuracy significantly increased with RF and $\mathrm{kNN}$, whereas the results of SVM were only slightly increased. It is indicated that the sample size and imbalanced data of training samples has more impact on the classification accuracy for KNN and RF than for SVM.

The highest accuracy for the three classifiers occurred when the training sample size was large enough, i.e., iset_5; $\mathrm{kNN}, \mathrm{RF}$, and $\mathrm{SVM}$ were $94.59 \%, 94.70 \%$, and $95.32 \%$, respectively (Figure 8). However, when the training sample sizes increased further (iset_6 and iset_7), the overall accuracy of the classifiers slightly decreased; kNN, RF, and SVM for iset_6 (iset_7) were 93.85\% (94.13\%), $94.32 \%(94.44 \%)$, and $95.12 \%(95.07 \%)$, respectively. It is suggested that if the training sample data is imbalanced between classes, the training sample sizes should be large enough to achieve the best performance of classifiers. If the training sample size is too large, it could change the proportion between classes, which lead to the decrease in overall accuracy. For all three classes in this study, 
the highest accuracies were achieved when the training sample size represented approximately $0.26 \%$ of the total study area. This is consistent with research of Colditz [58]; in which they stated that for the RF classifier, the training sample size should account for $0.25 \%$ of the total study area. Our results show that this case is not only valid for the RF classifier but also for the SVM and kNN classifiers.

The performance of Classifiers on different sample sizes

imbalanced datasets

Algorithm $\bigcirc \mathrm{kNN} \mathrm{RF} \mathrm{sVM}$

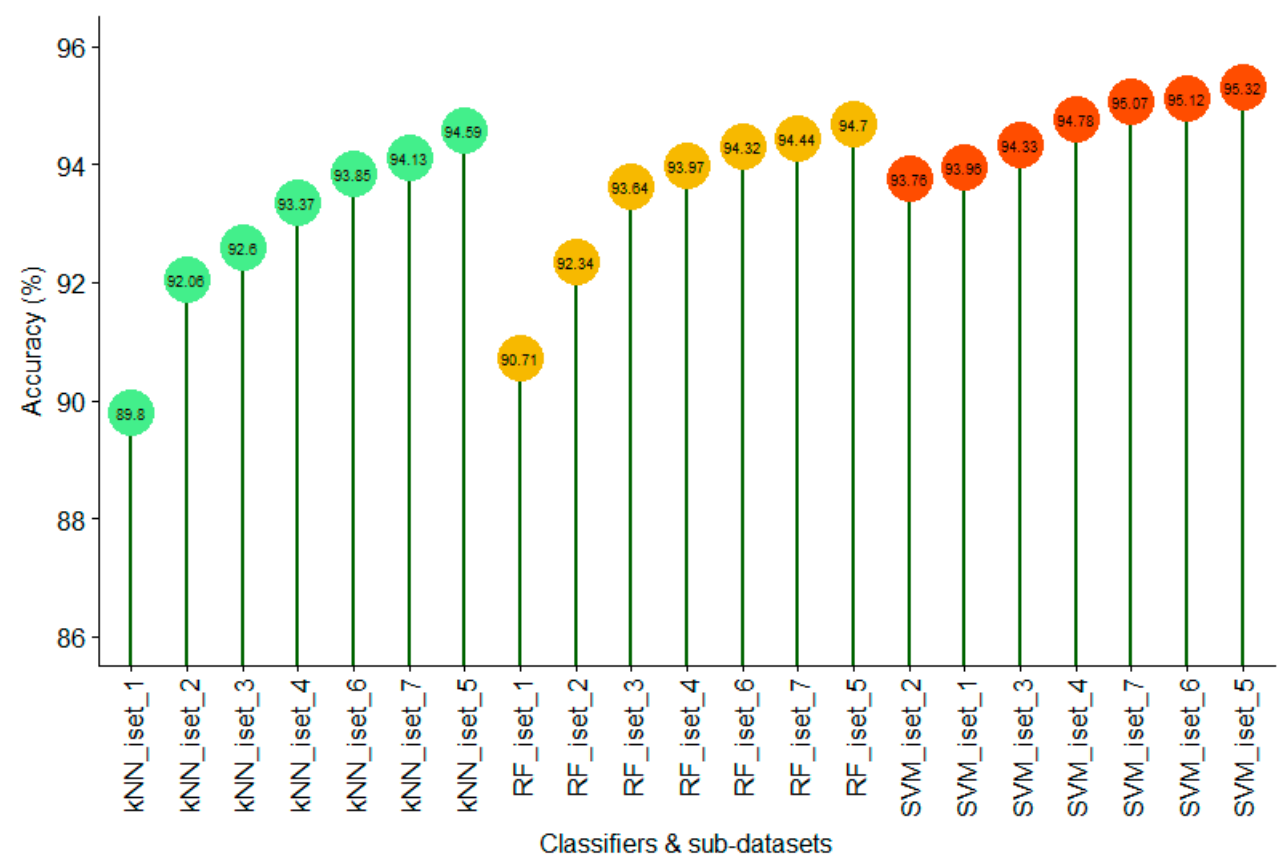

Figure 8. The performance of the $\mathrm{kNN}, \mathrm{SVM}$, and RF classifiers on different imbalanced training sample sizes.

\subsection{The Performance of Different Classifiers on Balanced Datasets}

For balanced datasets (bset_1 to bset_7), the SVM classifier still produced the highest accuracy at $95.29 \%$, followed by RF at $94.59 \%$ and $\mathrm{kNN}$ at $94.10 \%$. However, the performance of each classifier on different training sample sizes was only slightly different (Figure 9). For the kNN classifier with a small training sample size (bset_1 to bset_4), the training sample size had a strong impact on the accuracy of classification. The overall trend showed that the larger the training sample size, the higher the accuracy. Increasing the sample size from 50 pixels/class (bset_1) to 500 pixels/class (bset_4) resulted in an accuracy increase from $89.85 \%$ to $93.45 \%$. However, when the training sample size was high enough (more than 750 pixels /class), as it is for bset_5, bset_6, and bset_7, the classification accuracy was stable at $93.96 \%, 94.10 \%$, and $94.02 \%$, respectively.

With the RF classifier, the larger training sample also produced higher accuracy with the first 4 sub-datasets (bset_1 to bset_4). However, the difference at bset_1 and bset_4 was not as large as with the kNN classifier. With the smallest training sample (bset_1), RF produced a higher accuracy (91.47\%) than that of $\mathrm{kNN}(89.95 \%)$; however, with bset_4 (750 pixels/class), the accuracy results of RF and kNN were similar at $93.61 \%$ and $93.45 \%$, respectively. The stable accuracy results of bset_5, bset_6, and bset_7 (93.47\%,94.42\%, 94.59\%) were also observed with the RF classifier.

The SVM classifier showed different results. When the training sample size was small (bset_1 and bset_2), the classification accuracy was high and slightly different at $92.63 \%$ and $92.35 \%$, respectively. However, when the training sample size increased from 125 pixels/class (bset_2) to 250 pixels/class 
(bset_3) and 500 pixels/class (bset_4), the training sample size had a strong impact on classification accuracy. This is an interesting finding; because the results showed a contrast with previous studies which stated that the training sample size was less sensitive with SVM. This might be true if the training sample size is small enough (less than 125 pixels/class) or large enough (greater than 750 pixels/class). Figure 9 shows that when the balanced training sample size increasing from $0.20 \%$ (bset_5) to $0.33 \%$ (bset_7) of the total study area, the performance of the classifiers were similar between different training sample sizes. When comparing the three classifiers, SVM produced the highest accuracy, followed by RF and kNN.

\section{The performance of Classifiers on different sample sizes}

balanced datasets
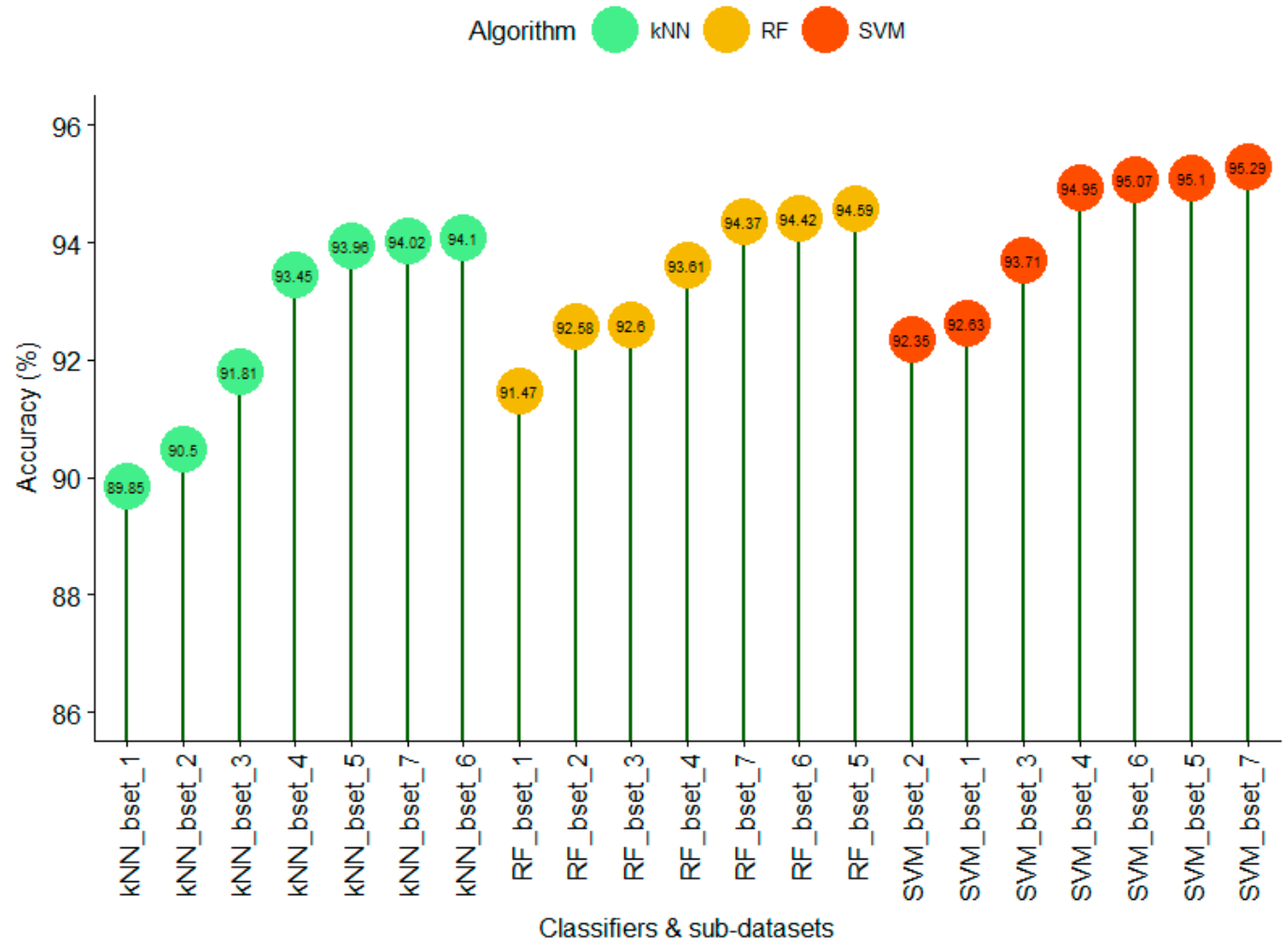

Figure 9. The performance of kNN, SVM, and RF classifiers on different balanced training sample sizes.

\section{Discussion}

Figure 10 shows the difference between OA of imbalanced and balanced sub-datasets from the 42 results of the 14 different training sample sizes for each classifier. Two different trends are clear: when training sample sizes were large (greater than or equal to 500 pixels/class) the performance of $\mathrm{kNN}$, $\mathrm{RF}$, and SVM on balanced and imbalanced datasets was not significantly different, except for kNN with dataset_5 (approximately 750 pixels/class).

Due to the actual proportion of land cover type on the landscape, the rare classes have a low number of pixels in the training sample (Table 2); therefore, in all sub-datasets (set_1 to set_7), the number of pixels in balanced datasets is always smaller than those of imbalanced sub-datasets. However, as mentioned earlier, when the training sample size is large enough, the performance of classifiers on balanced and imbalanced sub-datasets was similar. In other words, the classifiers are less sensitive to the imbalanced training data if the training sample size is large enough (i.e., greater than 750 pixels/class). 


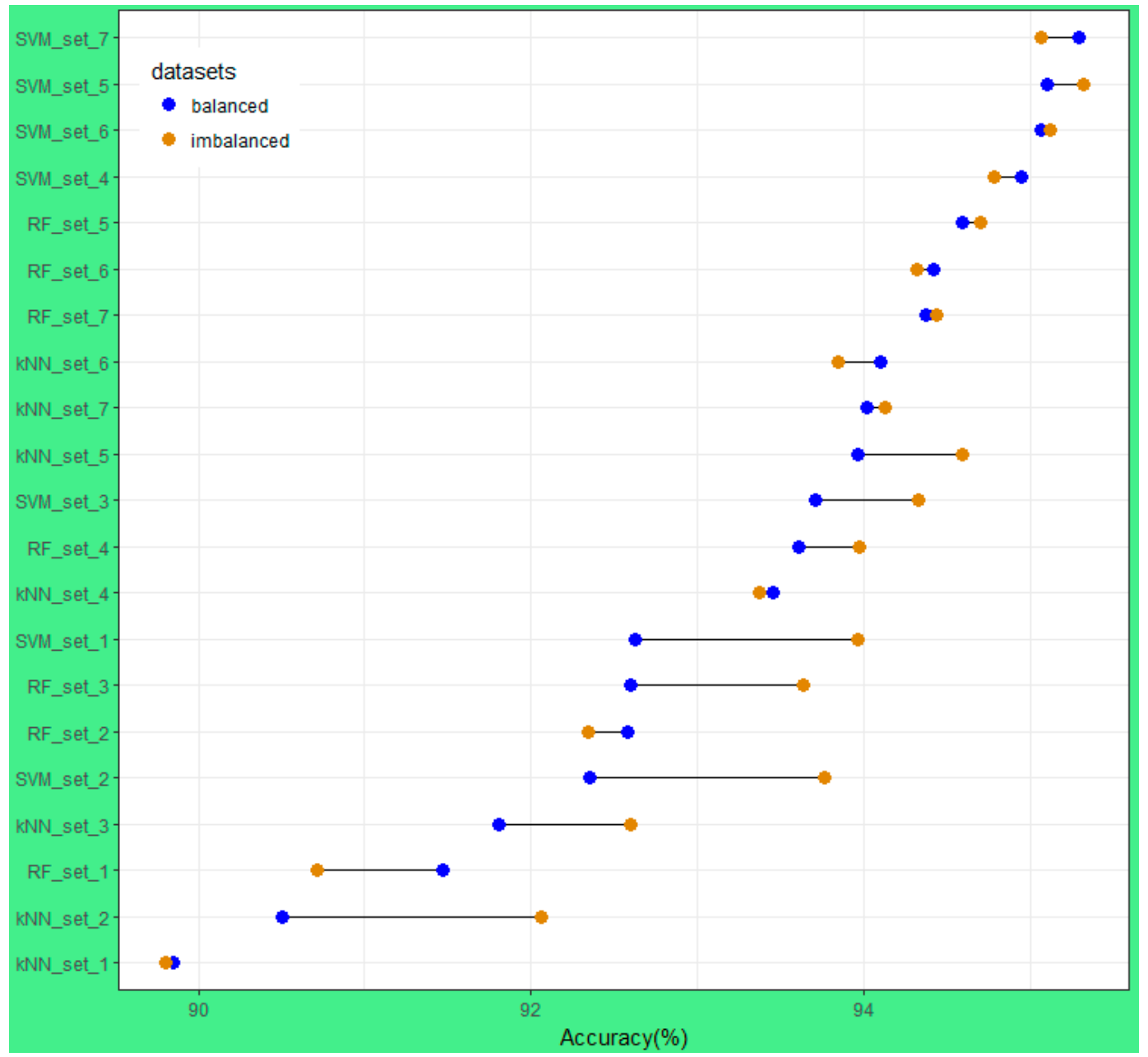

Figure 10. The difference in OA using balanced and imbalanced datasets.

Figure 10 shows that the performance of $\mathrm{kNN}$ at all sub-datasets for dataset_1, dataset_6, and dataset_7 was similar for balanced and imbalanced datasets, whereas the results of kNN on the remaining datasets were significantly different. It is indicated that the $\mathrm{kNN}$ classifier is less sensitive with imbalanced training sample data, although it varies with training sample sizes. With the RF and SVM classifiers, when training sample size is large enough (dataset_5, dataset_6, and dataset_7) the results of imbalanced and balanced were similar and very high (greater than $94.32 \%$ ).

Many studies investigated the performance of the RF classifier on different training sample sizes and strategies of different satellite images, but the conclusions are contradictory. Colditz [58] and Mellor et al. [59] found a trend for the area proportional allocation of training samples, in which the greater the land cover class area is the more training samples are needed to produce the best classification accuracy. In contrast, Dalponte et al. [60] and Jin et al. [61] stated that the RF classifier would perform better with balanced training sample data. Our results (Figure 9) show that if training sample size is small (less than 500 pixels/classes) the difference in accuracy of imbalanced and balanced data would be large (dataset-1, dataset-3), or small (dataset-2). However, when the training sample size is large enough (dataset-5, dataset-6, and dataset-7) the performance of RF on balanced and imbalanced training samples were similar. It must be mentioned that for those different conclusions, the RF classifier was used with different satellite imagery. Dalponte et al. [60] used HySpex-VNIR 1600 and HySpex-SWIR 320i data, Jin et al. [61] used Landsat imagery, Colditz [58] used MODIS imagery, and Mellor et al. [59] used Landsat TM composited with topographic and climate variables. Therefore, 
it is suggested that the performance of the RF classifier on different satellite imagery data with different training sample strategies (balanced versus imbalanced) is different.

With the SVM classifier, as shown in Figure 10 among the 42 classification results, the 8 highest accuracies belonged to the SVM classifier (SVM_set4, SVM_set5, SVM_set6, and SVM_set7). Particularly, the SVM classifier had the superior performance capability with small training sample sizes (set_1 and set_2 with approximately 50 pixels per class). SVM produced overall accuracies ranging from $93.76 \%$ to $93.96 \%$ (92.35\% to $92.63 \%$ ) for imbalanced (balanced) sub-datasets, compared to $89.80 \%$ to $92.06 \%$ (89.85\% to $90.50 \%$ ) and $90.71 \%$ to $92.34 \%$ (91.47\% to $92.58 \%$ ) for the $\mathrm{kNN}$ and $\mathrm{RF}$ classifiers, respectively. This is consistent with the study of Shao and Lunetta [62].

\section{Conclusions}

Classification of Sentinel-2 imagery (ten bands, $20 \mathrm{~m}$ ) using three different machine learning algorithms were implemented, evaluated, and compared. Fourteen different sub-datasets, including balanced and imbalanced, with different training sample sizes from 50 to more than 1250 pixels/class were performed. All classification results (OA) were high, approximately ranging from $90 \%$ to $95 \%$, with the SVM classifier on average producing the highest OA with the least sensitivity to training sample size, followed by the RF and kNN classifiers. The difference in OA between kNN and RF was large when the training sample size increased from sub-dataset- 1 to sub-dataset- 4 for both imbalanced and balanced cases; however, the difference between various training sample sizes of the SVM classifier was insignificant. For all three classifiers, when the training sample was large enough (greater than 750 pixels/class), with both imbalanced and balanced datasets (iset_5/bset_5, iset_6/bset_6, and iset_7/bset_7), the OA was approximately similar and high (over 93.85\%). Furthermore, it is recommended that in land cover classification using remote sensing images and machine learning algorithms, the training sample size should represent approximately $0.25 \%$ of the total study area.

Acknowledgments: We would like to acknowledge the Vietnamese government for its financial support. We are most grateful to Stefan Erasmi (Institute of Geography, University of Göttingen) for his helpful suggestions. This publication was supported financially by the Open Access Grant Program of the German Research Foundation (DFG) and the Open Access Publication Fund of the University of Göttingen. We also thank the anonymous reviewers for their valuable comments, which greatly improved our paper.

Author Contributions: Phan Thanh Noi analyzed the data, performed the experiments and computed the statistical analysis. Martin Kappas helped to conceive, design the statistical analysis and corrected the final manuscript. All authors developed and discussed the manuscript together and finally wrote the paper.

Conflicts of Interest: The authors declare no conflict of interest.

\section{Appendix A}
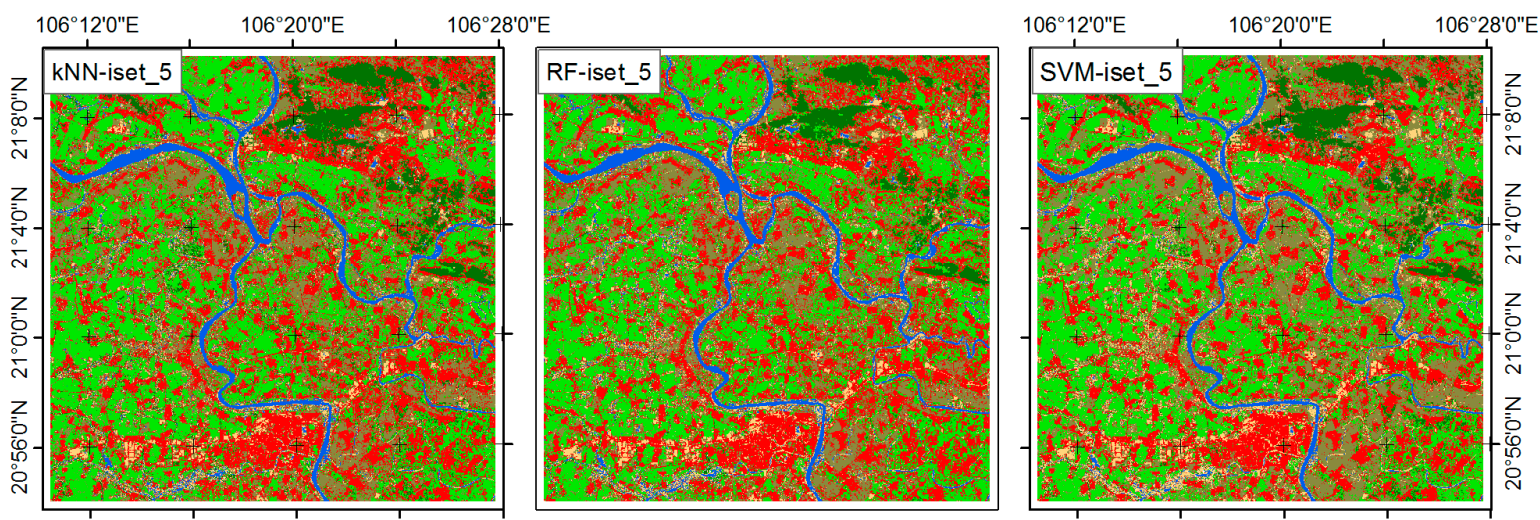

Figure A1. Cont. 


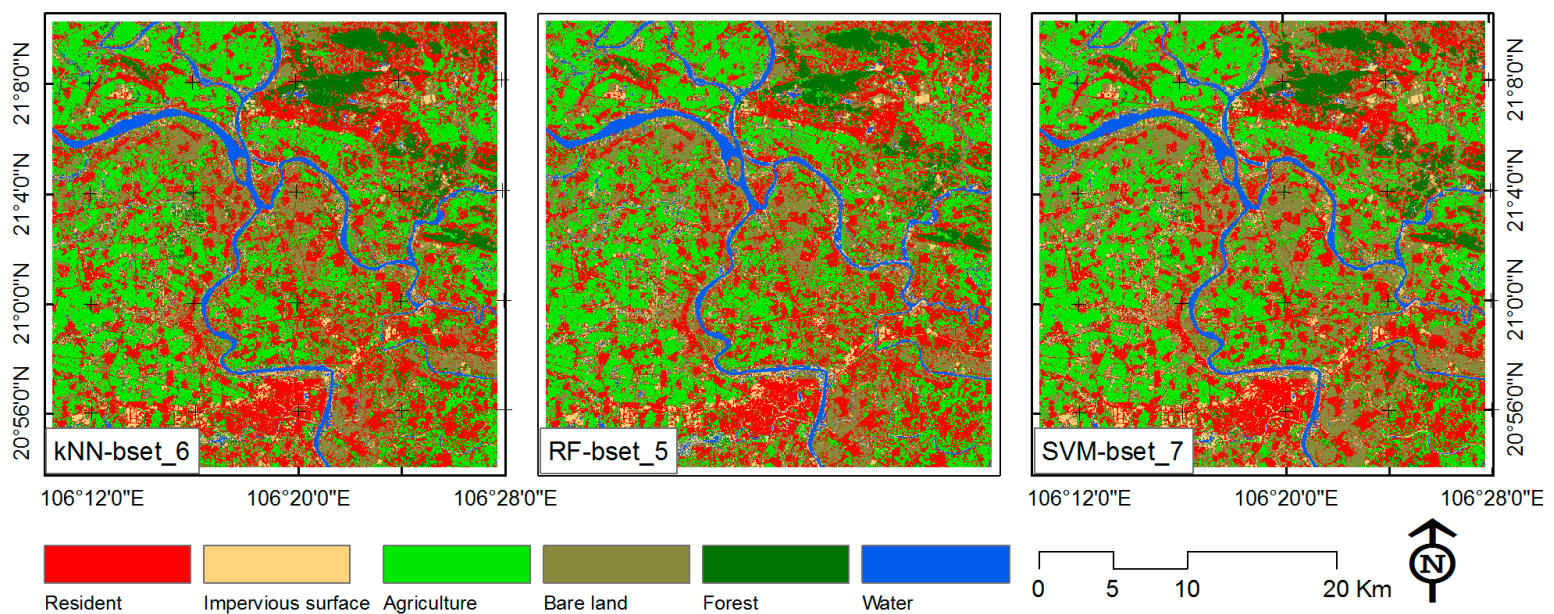

Figure A1. The classification results with the highest overall accuracy for each classifier.

\section{Appendix B}

Table A1. Overall Accuracy and 95\% confidence interval (error tolerance) of the probability estimate of all 42 classification results.

\begin{tabular}{|c|c|c|c|c|c|c|c|}
\hline Classifier & set1 & set2 & set3 & set4 & set5 & set6 & set7 \\
\hline RF_iset & $90.71 \pm 1.0$ & $.34 \pm 0.9$ & $93.64 \pm 0.8$ & $93.97 \pm 0.8$ & $94.70 \pm 0.7$ & $94.32 \pm 0.8$ & $94.44 \pm 0.8$ \\
\hline RF_bset & $91.47 \pm 0.9$ & $92.58 \pm 0.9$ & $92.60 \pm 0.9$ & $93.61 \pm 0.8$ & $94.59 \pm 0.7$ & $94.42 \pm 0.8$ & $94.37 \pm 0.8$ \\
\hline SVM_iset & $93.96 \pm 0.8$ & $93.76 \pm 0.8$ & $94.33 \pm 0.8$ & $94.78 \pm 0.7$ & $95.32 \pm 0.7$ & $95.12 \pm 0.7$ & $95.07 \pm 0.7$ \\
\hline SVM_bset & $92.63 \pm 0.9$ & $92.35 \pm 0.9$ & $93.71 \pm 0.8$ & $94.95 \pm 0.7$ & $95.10 \pm 0.7$ & $95.07 \pm 0.7$ & $95.29 \pm 0.7$ \\
\hline kNN_iset & $89.80 \pm 1.0$ & $92.06 \pm 0.9$ & $92.60 \pm 0.9$ & $93.37 \pm 0.8$ & $94.59 \pm 0.7$ & $93.85 \pm 0.8$ & $94.13 \pm 0.8$ \\
\hline kNN_bset & $89.85 \pm 1.0$ & $90.50 \pm 1.0$ & $91.81 \pm 0.9$ & $93.45 \pm 0.8$ & $93.96 \pm 0.8$ & $94.10 \pm 0.8$ & $94.02 \pm 0.8$ \\
\hline
\end{tabular}

\section{References}

1. DeFries, R.S.; Foley, J.A.; Asner, G.P. Land-use choices: Balancing human needs and ecosystem function. Front. Ecol. Environ. 2004, 2, 249-257. [CrossRef]

2. Foley, J.A.; DeFries, R.; Asner, G.P.; Barford, C.; Bonan, G.; Carpenter, S.R.; Chapin, F.S.; Coe, M.T.; Daily, G.C.; Gibbs, H.K.; et al. Global consequences of land use. Science 2005, 309, 570-574. [CrossRef] [PubMed]

3. Verburg, P.H.; Neumann, K.; Nol, L. Challenges in using land use and land cover data for global change studies. Glob. Chang. Biol. 2011, 17, 974-989. [CrossRef]

4. Hansen, T. A review of large area monitoring of land cover change using Landsat data. Remote Sens. Environ. 2012, 122, 66-74. [CrossRef]

5. Wessels, K.J.; Reyers, B.; Jaarsveld, A.S.; Rutherford, M.C. Identification of potential conflict areas between land transformation and biodiversity conservation in north-eastern South Africa. Agric. Ecosyst. Environ. 2003, 95, 157-178. [CrossRef]

6. Fry, J.; Xian, G.Z.; Jin, S.; Dewitz, J.; Homer, C.G.; Yang, L.; Barnes, C.A.; Herold, N.D.; Wickham, J.D. Completion of the 2006 national land cover database for the conterminous United States. Photogramm. Eng. Remote Sens. 2011, 77, 858-864.

7. Burkhard, B.; Kroll, F.; Nedkov, S.; Müller, F. Mapping ecosystem service supply, demand and budgets. Ecol. Indic. 2012, 21, 17-29. [CrossRef]

8. Gebhardt, S.; Wehrmann, T.; Ruiz, M.A.M.; Maeda, P.; Bishop, J.; Schramm, M.; Kopeinig, R.; Cartus, O.; Kellndorfer, J.; Ressl, R.; et al. MAD-MEX: Automatic wall-to-wall land cover monitoring for the Mexican REDD-MRV program using all Landsat data. Remote Sens. 2014, 6, 3923-3943. [CrossRef] 
9. Guidici, D.; Clark, M.L. One-Dimensional convolutional neural network land-cover classification of multi-seasonal hyperspectral imagery in the San Francisco Bay Area, California. Remote Sens. 2017, 9, 629. [CrossRef]

10. Topaloğlu, H.R.; Sertel, E.; Musaoğlu, N. Assessment of classification accuracies of SENTINEL-2 and LANDSAT-8 data for land cover/use mapping. In International Archives of the Photogrammetry, Remote Sensing and Spatial Information Sciences; ISPRS: Prague, Czech Republic, 2016; Volume XLI-B8, pp. 1055-1059.

11. Khatami, R.; Mountrakis, G.; Stehman, S.V. A meta-analysis of remote sensing research on supervised pixel-based land cover image classification processes: General guidelines for practitioners and future research. Remote Sens. Environ. 2016, 177, 89-100. [CrossRef]

12. Duro, D.C.; Franklin, S.E.; Dubé, M.G. A comparison of pixel-based and object-based image analysis with selected machine learning algorithms for the classification of agricultural landscapes using SPOT-5 HRG imagery. Remote Sens. Environ. 2012, 118, 259-272. [CrossRef]

13. Xia, J.S.; Mura, M.D.; Chanussot, J.; Du, P.; He, X. Random subspace ensembles for hyperspectral image classification with extended morphological attribute profiles. IEEE Trans. Geosci. Remote Sens. 2015, 53, 4768-4786. [CrossRef]

14. Chen, Y.; Dou, P.; Yang, X. Improving land use/cover classification with a multiple classifier system using AdaBoost integration technique. Remote Sens. 2017, 9, 1055. [CrossRef]

15. Gomez, C.; White, J.C.; Wulder, M.A. Optical remotely sensed time series data for land cover classification: A review. Int. Soc. Photogramm. 2016, 116, 55-72. [CrossRef]

16. Martins, V.S.; Barbosa, C.C.F.; de Carvalho, L.A.S.; Jorge, D.S.F.; Lobo, F.L.; Novo, E.M.L.M. Assessment of atmospheric correction methods for Sentinel-2 MSI images applied to Amazon floodplain lakes. Remote Sens. 2017, 9, 322. [CrossRef]

17. Wang, Q.; Blackburn, G.A.; Onojeghuo, A.O.; Dash, J.; Zhou, L.; Zhang, Y.; Atkinson, P.M. Fusion of Landsat 8 OLI and Sentinel-2 MSI data. IEEE Trans. Geosci. Remote Sens. 2017, 55, 3885-3899. [CrossRef]

18. Gao, Q.; Zribi, M.; Escorihuela, M.J.; Baghdadi, N. Synergetic use of Sentinel-1 and Sentinel-2 data for soil moisture mapping at $100 \mathrm{~m}$ resolution. Sensors 2017, 17, 1966. [CrossRef] [PubMed]

19. Yang, X.; Zhao, S.; Qin, X.; Zhao, N.; Liang, L. Mapping of urban surface water bodies from Sentinel-2 MSI imagery at $10 \mathrm{~m}$ resolution via NDWI-based image sharpening. Remote Sens. 2017, 9, 596. [CrossRef]

20. Eitel, J.U.; Vierling, L.A.; Litvak, M.E.; Long, D.S.; Schulthess, U.; Ager, A.A.; Krofcheck, D.J.; Stoscheck, L. Broadband red-edge information from satellites improves early stress detection in a New Mexico conifer woodland. Remote Sens. Environ. 2011, 115, 3640-3646. [CrossRef]

21. Sibanda, M.; Mutanga, O.; Rouget, M. Examining the potential of Sentinel-2 MSI spectral resolution in quantifying above ground biomass across different fertilizer treatments. ISPRS J. Photogramm. Remote Sens. 2015, 110, 55-65. [CrossRef]

22. Clevers, J.G.P.W.; Kooistra, L.; van den Brande, M.M.M. Using Sentinel-2 data for retrieving LAI and leaf and canopy chlorophyll content of a potato crop. Remote Sens. 2017, 9, 405. [CrossRef]

23. Pesaresi, M.; Corbane, C.; Julea, A.; Florczyk, A.J.; Syrris, V.; Soille, P. Assessment of the added-value of Sentinel-2 for detecting built-up areas. Remote Sens. 2016, 8, 299. [CrossRef]

24. Lefebvre, A.; Sannier, C.; Corpetti, T. Monitoring urban areas with Sentinel-2A data: Application to the update of the copernicus high resolution layer imperviousness degree. Remote Sens. 2016, 8, 606. [CrossRef]

25. Lu, D.; Weng, Q.A. Survey of image classification methods and techniques for improving classification performance. Int. J. Remote Sens. 2007, 28, 823-870. [CrossRef]

26. Friedl, M.A.; Brodley, C.E. Decision tree classification of land cover from remotely sensed data. Remote. Sens. Environ. 1997, 61, 399-409. [CrossRef]

27. Waske, B.; Braun, M. Classifier ensembles for land cover mapping using multitemporal SAR imagery. ISPRS J. Photogramm. Remote Sens. 2009, 64, 450-457. [CrossRef]

28. Li, C.; Wang, J.; Wang, L.; Hu, L.; Gong, P. Comparison of classification algorithms and training sample sizes in urban land classification with Landsat Thematic Mapper imagery. Remote Sens. 2014, 6, 964-983. [CrossRef]

29. Jhonnerie, R.; Siregar, V.P.; Nababan, B.; Prasetyo, L.B.; Wouthuyzen, S. Random forest classification for mangrove land cover mapping using Landsat 5 TM and Alos Palsar imageries. Procedia Environ. Sci. 2015, 24, 215-221. [CrossRef] 
30. Basukala, A.K.; Oldenburg, C.; Schellberg, J.; Sultanov, M.; Dubovyk, O. Towards improved land use mapping of irrigated croplands: Performance assessment of different image classification algorithms and approaches. Eur. J. Remote. Sens. 2017, 50, 187-201. [CrossRef]

31. Prasad, A.; Iverson, L.; Liaw, A. Newer classification and regression tree techniques: Bagging and random forests for ecological prediction. Ecosystems 2006, 9, 181-199. [CrossRef]

32. Naidoo, L.; Cho, M.A.; Mathieu, R.; Asner, G. Classification of savanna tree species, in the Greater Kruger National Park region, by integrating hyperspectral and LiDAR data in a random forest data mining environment. ISPRS J. Photogramm. Remote Sens. 2012, 69, 167-179. [CrossRef]

33. Breiman, L. Random forests. Mach. Learn. 2001, 45, 5-32. [CrossRef]

34. Adam, E.; Mutanga, O.; Odindi, J.; Abdel-Rahman, E.M. Land-use/cover classification in a heterogeneous coastal landscape using RapidEye imagery: Evaluating the performance of random forest and support vector machines classifiers. Int. J. Remote Sens. 2014, 35, 3440-3458. [CrossRef]

35. Ghosh, A.; Joshi, P.K. A comparison of selected classification algorithms for mapping bamboo patches in lower Gangetic plains using very high resolution WorldView 2 imagery. Int. J. Appl. Earth Obs. Geoinf. 2014, 26, 298-311. [CrossRef]

36. Pouteaua, R.; Collinb, A.; Stolla, B. A Comparison of Machine Learning Algorithms for Classification of Tropical Ecosystems Observed by Multiple Sensors at Multiple Scales; International Geoscience and Remote Sensing Symposium: Vancouver, BC, Canada, 2011.

37. Heydari, S.S.; Mountrakis, G. Effect of classifier selection, reference sample size, reference class distribution and scene heterogeneity in per-pixel classification accuracy using 26 Landsat sites. Remote Sens. Environ. 2018, 204, 648-658. [CrossRef]

38. U.S. Geological Survey. Available online: https:/ / earthexplorer.usgs.gov / (accessed on 22 July 2017).

39. Louis, J.; Debaecker, V.; Pflug, B.; Main-Knorn, M.; Bieniarz, J.; Mueller-Wilm, U.; Cadau, E.; Gascon, F. Sentinel-2 Sen2Cor: L2A Processor for Users. In Proceedings of the Living Planet Symposium (Spacebooks Online), Prague, Czech Republic, 9-13 May 2016; pp. 1-8.

40. Kuhn, M. Building predictive models in R using the caret package. J. Stat. Softw. 2008, 28, 1-26. [CrossRef]

41. Qian, Y.; Zhou, W.; Yan, J.; Li, W.; Han, L. Comparing machine learning classifiers for object-based land cover classification using very high resolution imagery. Remote Sens. 2015, 7, 153-168. [CrossRef]

42. Knorn, J.; Rabe, A.; Radeloff, V.C.; Kuemmerle, T.; Kozak, J.; Hostert, P. Land cover mapping of large areas using chain classification of neighboring Landsat satellite images. Remote. Sens. Environ. 2009, 113, 957-964. [CrossRef]

43. Shi, D.; Yang, X. Support vector machines for land cover mapping from remote sensor imagery. In Monitoring and Modeling of Global Changes: A Geomatics Perspective; Springer: Dordrecht, The Netherlands, 2015; pp. 265-279.

44. Ballanti, L.; Blesius, L.; Hines, E.; Kruse, B. Tree species classification using hyperspectral imagery: A comparison of two classifiers. Remote Sens. 2016, 8, 445. [CrossRef]

45. Exelis Visual Information Solutions. ENVI Help; Exelis Visual Information Solutions: Boulder, CO, USA, 2013.

46. Melgani, F.; Bruzzone, L. Classification of hyperspectral remote sensing images with support vector machines. IEEE Trans. Geosci. Remote Sens. 2004, 42, 1778-1790. [CrossRef]

47. Huang, C.; Davis, L.S.; Townshend, J.R.G. An assessment of support vector machines for land cover classification. Int. J. Remote Sens. 2002, 23, 725-749. [CrossRef]

48. Liaw, A.; Wiener, M. Classification and regression by randomForest. R News 2002, 2, 18-22.

49. Immitzer, M.; Atzberger, C.; Koukal, T. Tree species classification with random forest using very high spatial resolution 8-Band WorldView-2 satellite data. Remote Sens. 2012, 4, 2661-2693. [CrossRef]

50. Zhang, H.K.; Roy, D.P. Using the $500 \mathrm{~m}$ MODIS land cover product to derive a consistent continental scale $30 \mathrm{~m}$ Landsat land cover classification. Remote Sens. Environ. 2017, 197, 15-34. [CrossRef]

51. Feng, Q.; Liu, J.; Gong, J. UAV remote sensing for urban vegetation mapping using random forest and texture analysis. Remote Sens. 2015, 7, 1074-1094. [CrossRef]

52. Duda, R.; Hart, P. Pattern Classification and Scene Analysis; John Wiley \& Sons: New York, NY, USA, 1973.

53. Franco-Lopez, H.; Ek, A.R.; Bauer, M.E. Estimation and mapping of forest stand density, volume and cover type using the k-Nearest Neighbors method. Remote Sens. Environ. 2001, 77, 251-274. [CrossRef]

54. Akbulut, Y.; Sengur, A.; Guo, Y.; Smarandache, F. NS-k-NN: Neutrosophic Set-Based k-Nearest Neighbors classifier. Symmetry 2017, 9, 179. [CrossRef] 
55. Wei, C.; Huang, J.; Mansaray, L.R.; Li, Z.; Liu, W.; Han, J. Estimation and mapping of winter oilseed rape LAI from high spatial resolution satellite data based on a hybrid method. Remote Sens. 2017, 9, 488. [CrossRef]

56. He, H.; Garcia, E.A. Learning from imbalanced data. IEEE Trans. Knowl. Data Eng. 2009, 21, 1263-1284.

57. Baraldi, A.; Puzzolo, V.; Blonda, P.; Bruzzone, L.; Tarantino, C. Automatic spectral rule-based preliminary mapping of calibrated Landsat TM and ETM+ images. IEEE Trans. Geosci. Remote Sens. 2006, 44, 2563-2586. [CrossRef]

58. Colditz, R.R. An evaluation of different training sample allocation schemes for discrete and continuous land cover classification using decision tree-based algorithms. Remote Sens. 2015, 7, 9655-9681. [CrossRef]

59. Mellor, A.; Boukir, S.; Haywood, A.; Jones, S. Exploring issues of training data imbalance and mislabelling on random forest performance for large area land cover classification using the ensemble margin. ISPRS J. Photogramm. Remote Sens. 2015, 105, 155-168. [CrossRef]

60. Dalponte, M.; Orka, H.O.; Gobakken, T.; Gianelle, D.; Naesset, E. Tree species classification in boreal forests with hyperspectral data. IEEE Trans. Geosci. Remote Sens. 2013, 51, 2632-2645. [CrossRef]

61. Jin, H.; Stehman, S.V.; Mountrakis, G. Assessing the impact of training sample extraction on accuracy of an urban classification: A case study in Denver, Colorado. Int. J. Remote Sens. 2014, 35, 2067-2081.

62. Shao, Y.; Lunetta, R.S. Comparison of support vector machine, neural network, and CART algorithms for the land-cover classification using limited training data points. ISPRS J. Photogramm. Remote Sens. 2012, 70, 78-87. [CrossRef]

(C) 2017 by the authors. Licensee MDPI, Basel, Switzerland. This article is an open access article distributed under the terms and conditions of the Creative Commons Attribution (CC BY) license (http:/ / creativecommons.org/licenses/by/4.0/). 